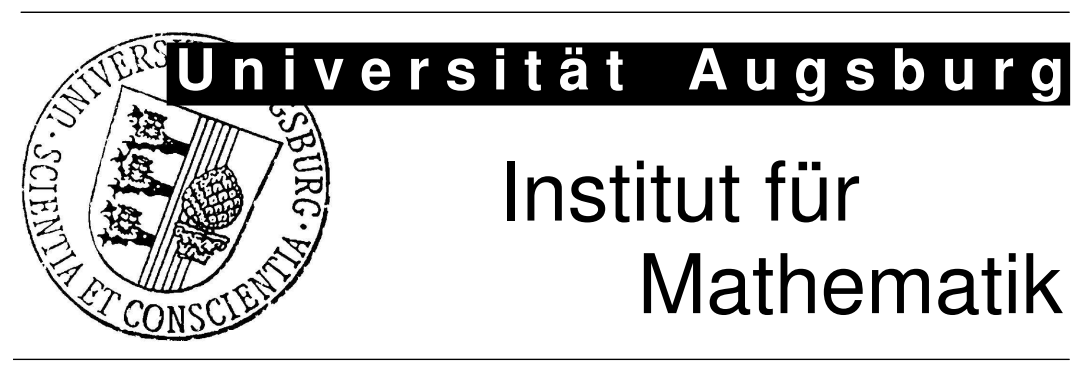

Christian Bräu, Lothar Heinrich

Multivariate Poisson Distributions Associated with Boolean Models 


\section{Impressum:}

\section{Herausgeber:}

Institut für Mathematik

Universität Augsburg

86135 Augsburg

http://www . math. uni-augsburg.de/de/forschung/preprints.html

\section{ViSdP:}

Lothar Heinrich

Institut für Mathematik

Universität Augsburg

86135 Augsburg

Preprint: Sämtliche Rechte verbleiben den Autoren (C) 2015 


\title{
Multivariate Poisson Distributions Associated with Boolean Models
}

\author{
Christian Bräu and Lothar Heinrich ${ }^{1}$
}

\begin{abstract}
We consider a $d$-dimensional Boolean model $\Xi=\left(\Xi_{1}+X_{1}\right) \cup\left(\Xi_{2}+X_{2}\right) \cup \cdots$ generated by a Poisson point process $\left\{X_{i}, i \geq 1\right\}$ with intensity measure $\Lambda$ and a sequence $\left\{\Xi_{i}, i \geq 1\right\}$ of independent copies of some random compact set $\Xi_{0}$. Given compact sets $K_{1}, \ldots, K_{\ell}$, we show that the discrete random vector $\left(N\left(K_{1}\right), \ldots, N\left(K_{\ell}\right)\right)$, where $N\left(K_{j}\right)$ equals the number of shifted sets $\Xi_{i}+X_{i}$ hitting $K_{j}$, obeys a $\ell$-variate Poisson distribution with $2^{\ell}-1$ parameters. We obtain explicit formulae for all these parameters which can be estimated consistently from an observation of the union set $\Xi$ in some unboundedly expanding window $W_{n}($ as $n \rightarrow \infty)$ provided that the Boolean model is stationary. Some of these results can be extended to unions of Poisson $k$-cylinders for $1 \leq k<d$ and more general set-valued functionals of independently marked Poisson processes.

Keywords : RANDom Closed SETs, indePEndently marked Poisson Process, GenERATING FUNCTIONAL, MULTIVARIATE PROBABILITY GENERATING FUNCTION, HIGHERORDER COVARIANCES, EMPIRICAL VOLUME FRACTION
\end{abstract}

AMS 2010 MSC : Primary: 60D05, 60E05; SEcondary: 60E10, 62F10

\section{Introduction and preliminaries}

The Boolean model (briefly BM; also known as Poisson grain or Poisson blob model) is one of the best studied and most frequently used models to describe two-phase systems of random sets which decompose the $d$-dimensional Euclidean space $\mathbb{R}^{d}$ into a vacant region (white) and a region consisting of irregularly shaped clumps (black), see Fig.1. This clumping structure is generated by unions of completely randomly distributed independent random compact sets,

\footnotetext{
${ }^{1}$ Corresponding author: Institute of Mathematics, Augsburg University, 86135 Augsburg, Germany E-mail: heinrich@math.uni-augsburg.de
} 


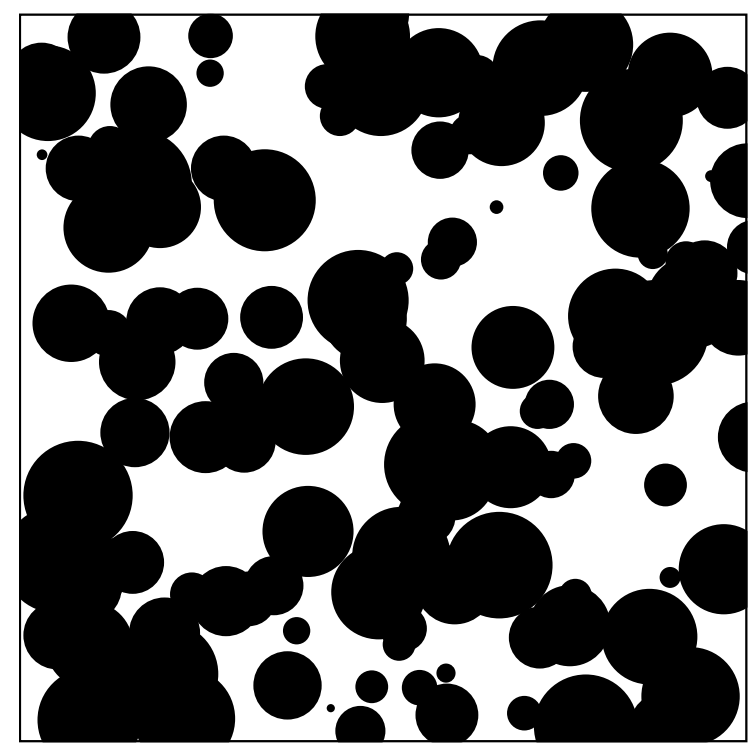

Figure 1: Realization of a 2D-Boolean model with discs

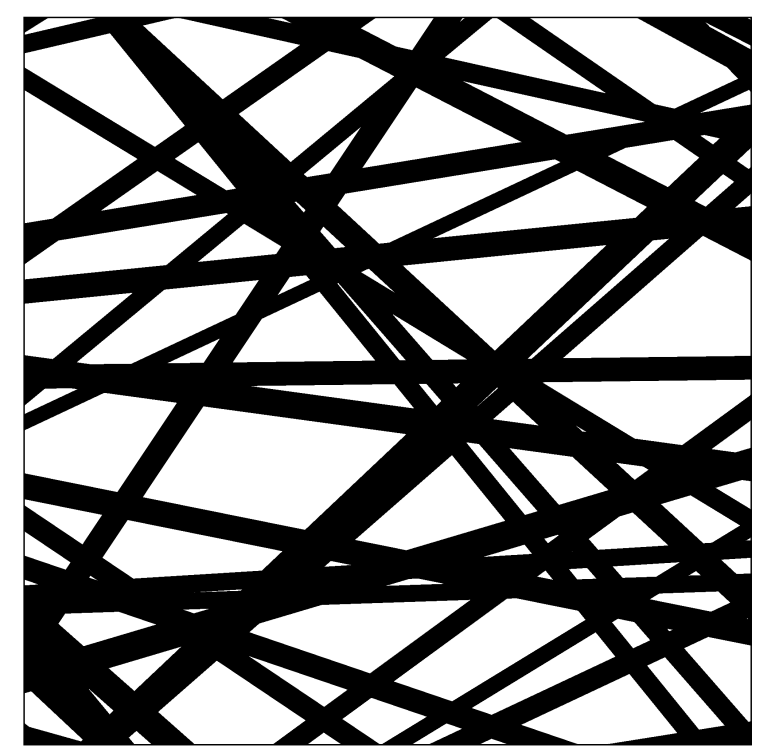

Figure 2: Realization of a 2D-Poisson strip process

see e.g. [12], [4], [13],[16],[2] for more details. To be precise, we first recall the definition of a (not necessarily stationary) $\mathrm{BM} \Xi=\Xi_{\Lambda, Q}$ as countable union set

$$
\Xi_{\Lambda, Q}=\bigcup_{i \geq 1}\left(\Xi_{i}+X_{i}\right)
$$

of independent copies $\Xi_{1}, \Xi_{2}, \ldots$ of a generic random compact set $\Xi_{0}$ (called typical grain) with distribution $Q$ (on the Borel $\sigma$-algebra $\mathcal{B}\left(\mathcal{K}^{d}\right)$, where $\mathcal{K}^{d}$ denotes the metric space of non-empty compact sets in $\mathbb{R}^{d}$ equipped with the Hausdorff metric), where the $\Xi_{i}$ 's are independently shifted by the atoms of the Poisson point process $\Pi_{\Lambda}=\sum_{i \geq 1} \delta_{X_{i}}$ with locally finite intensity measure $\Lambda$ on the Borel $\sigma$-algebra $\mathcal{B}\left(\mathbb{R}^{d}\right)$. For notational ease, each atom of the locally finite random counting measure $\Pi_{\Lambda}$ occurs in the sum $\sum_{i \geq 1}$ resp. in the union $\bigcup_{i \geq 1}$ according to its multiplicity, where $\Pi_{\Lambda}$ has multiple atoms iff $\Lambda$ has atoms, see [16], p. 59. By definition of $\Pi_{\Lambda}$, the random numbers $\Pi_{\Lambda}\left(B_{1}\right), \ldots, \Pi_{\Lambda}\left(B_{\ell}\right)$ are mutually independent if the bounded sets $B_{1}, \ldots, B_{\ell} \in \mathcal{B}\left(\mathbb{R}^{d}\right)$ are pairwise disjoint and $\Pi_{\Lambda}\left(B_{j}\right)$ is Poisson distributed with mean $\Lambda\left(B_{j}\right)$ for $j=1, \ldots, \ell$. The stationarity of $\Pi_{\Lambda}$ and thus the stationarity of the BM (1.1) is equivalent with the shift-invariance of the intensity measure $\Lambda$ and this in turn means that $\Lambda(\cdot)=\lambda|\cdot|_{d}$ with some constant $\lambda>0$ (called intensity of the BM) and Lebesgue measure $|\cdot|_{d}$ on $\mathcal{B}\left(\mathbb{R}^{d}\right)$. In what follows, $\Xi_{Q}^{\lambda}$ will denote a stationary BM with intensity $\lambda>0$.

The random union set $\Xi_{\Lambda, Q}$ defined by (1.1) over some probability space $[\Omega, \mathcal{F}, \mathbf{P}]$ (which always exists) need not to be closed $\mathbf{P}$-a.s., see e.g. [7] for counterexamples. The (P-a.s.) 
closedness of the BM (1.1) is guaranteed iff, for any $r>0$,

$$
0<\int_{\mathbb{R}^{d}} Q\left(\left\{K \in \mathcal{K}^{d}:(K+x) \cap B_{r}^{d} \neq \emptyset\right\}\right) \Lambda(\mathrm{d} x)=\mathbf{E} \Lambda\left(B_{r}^{d} \oplus\left(-\Xi_{0}\right)\right)<\infty,
$$

where $B_{r}^{d}:=\left\{x \in \mathbb{R}^{d}:\|x\| \leq r\right\}$ and $A \oplus B:=\{a+b: a \in A, b \in B\}$, see [12] and [5] as special case of closedness conditions for general grain-germ models.

Under condition (1.2) the random variable

$$
N(K)=\#\left\{i \geq 1: K \cap\left(\Xi_{i}+X_{i}\right) \neq \emptyset\right\}
$$

taking values in $\mathbb{Z}_{+}=\{0,1,2, \ldots\}$ is (as consequence of our model assumptions) Poisson distributed with finite mean $\mathbf{E} \Lambda\left(K \oplus\left(-\Xi_{0}\right)\right)>0$ for each $K \in \mathcal{K}^{d}$. In this paper we are interested in the distribution of the $\mathbb{Z}_{+}^{\ell}$-valued random vector $\left(N\left(K_{1}\right), \ldots, N\left(K_{\ell}\right)\right)$ for fixed pairwise different, compact sets $K_{1}, \ldots, K_{\ell}$ in $\mathbb{R}^{d}$ and any $\ell \geq 2$. Obviously, the components $N\left(K_{i}\right)$ are correlated random variables unless $\Xi_{0}$ is bounded and the $K_{i}$ 's are specially chosen. In [1] the special case of two distinct points $x, y \in \mathbb{R}^{d}$ has been treated by a rather lengthy explicit computation of the probabilities $\mathbf{P}(N(\{x\})=i, N(\{y\})=j)$ for $i, j=0,1, \ldots$ In Sect. 2 we extend this result in two ways, namely, we consider more than two points $x_{1}, \ldots, x_{\ell}$ and can even replace the $x_{i}$ 's by compact sets $K_{i}$. To avoid the awfully long computation of the joint probabilities of $\left\{N\left(K_{i}\right)=n_{i}\right\}, i=1, \ldots, \ell$, we derive instead in Theorem 2.1 the shape of the probability generating function (short: PGF)

$$
\mathbf{E}\left[z_{1}^{N\left(K_{1}\right)} \cdots z_{\ell}^{N\left(K_{\ell}\right)}\right]=\sum_{n_{1}, \ldots, n_{\ell} \geq 0} z_{1}^{n_{1}} \cdots z_{\ell}^{n_{\ell}} \mathbf{P}\left(N\left(K_{1}\right)=n_{1}, \ldots, N\left(K_{\ell}\right)=n_{\ell}\right)
$$

for $z_{1}, \ldots, z_{\ell} \in \mathbb{C}^{1}$ satisfying $\max _{1 \leq i \leq \ell}\left|z_{i}\right| \leq 1$.

It turns out that $\left(N\left(K_{1}\right), \ldots, N\left(K_{\ell}\right)\right)$ possesses an $\ell$-variate Poisson distribution. Since there exist different multivariate extensions of the Poisson distribution, see e.g. [9], we recall the definition of that $\ell$-variate Poisson distribution which seems to be most meaningful not only in our situation. A random vector $\left(N_{1}, \ldots, N_{\ell}\right)$ with values in $\mathbb{Z}_{+}^{\ell}$ is said to be $\ell$-variate Poisson distributed if its PGF $g\left(z_{1}, \ldots, z_{\ell}\right)=\mathbf{E}\left[z_{1}^{N_{1}} \cdots z_{\ell}^{N_{\ell}}\right]$ possesses the form

$$
g\left(z_{1}, \ldots, z_{\ell}\right):=\exp \left\{\sum_{\emptyset \neq J \subseteq L} \mu_{J} \prod_{j \in J}\left(z_{j}-1\right)\right\}=\exp \left\{\gamma_{\emptyset}+\sum_{\emptyset \neq J \subseteq L} \gamma_{J} \prod_{j \in J} z_{j}\right\}
$$

for all $z_{1}, \ldots, z_{\ell} \in \mathbb{C}^{1}$ with positive expectations $\mu_{i}=\mathbf{E} N_{i}$ and further $2^{\ell}-1-\ell$ parameters $\mu_{J} \geq 0$ for $J \subseteq L:=\{1, \ldots, \ell\}, \# J=2, \ldots, \ell$, where $\mu_{J}$ coincides with the mixed (factorial) 
cumulant of the subvector $\left(N_{j}\right)_{j \in J}$ which have to satisfy the consistency conditions

$$
\gamma_{\emptyset}:=\sum_{\emptyset \neq J \subseteq L}(-1)^{\# J} \mu_{J}<0 \text { and } \gamma_{I}:=\sum_{I \subseteq J \subseteq L}(-1)^{\#(J \backslash I)} \mu_{J} \geq 0 \text { for } \emptyset \neq I \subseteq L .
$$

The conditions (1.6) are necessary (and sufficient) for a non-degenerate $\ell$-variate Poisson distribution since (1.5) implies the relations $\frac{\partial^{\# I}}{\left(\partial z_{i}\right)_{i \in I}} \log g\left(z_{1}, \ldots, z_{\ell}\right) \geq 0$ for $I \subseteq L$ and all $z_{1}, \ldots, z_{\ell} \in[0,1]$ and $\gamma_{\emptyset}=-\sum_{\emptyset \neq I \subseteq L} \gamma_{I}$. Various structural properties of this multivariate Poisson distribution are known : (i) its existence as limit of multivariate binomial distributions, see [10], (ii) its characterization by recurrence relations of the density functions $\mathbf{P}\left(N_{1}=n_{1}, \ldots, N_{\ell}=n_{\ell}\right)$, see [11] and (iii) it is the only infinitely divisible distribution which is marginally Poisson, see [3] and [9]. It is noteworthy to mention that $\mu_{i j}=\operatorname{Cov}\left(N_{i}, N_{j}\right)=0$ for $i \neq j$ implies independence of the components $N_{i}$ and $N_{j}$. On the other hand, $\mu_{i j}=0$ for all pairs $i, j \in L, i \neq j, \# L \geq 3$, does not necessarily imply neither $\mu_{L}=0$ nor mutual independence of the components $N_{1}, \ldots, N_{\ell}$. The rest of this paper is organized as follows: In the next Sect. 2 we formulate and prove the announced main result for BM's. In Sect. 3 we shall discuss the parameters in case of a stationary BM and derive strongly consistent estimators for all parameters occurring in the PGF (2.7) based on an single observation of the union set (1.1) in an unboundedly increasing sampling window $W_{n}$. In the final Sect. 4 we extend Theorem 2.1 to other random set models driven by independently marked Poisson processes.

\section{Main result}

Theorem 2.1. Let $\Xi_{\Lambda, Q}$ be a BM as defined by (1.1) satisfying (1.2). Then the PGF of the random vector $\left(N\left(K_{1}\right), \ldots, N\left(K_{\ell}\right)\right)$ with components defined by $(1.3)$ for fixed $K_{1}, \ldots, K_{\ell} \in \mathcal{K}^{d}$ such that $\min _{1 \leq i \leq \ell} \mathbf{E} \Lambda\left(K_{i} \oplus\left(-\Xi_{0}\right)\right)>0$ takes the form

$$
\mathbf{E}\left[z_{1}^{N\left(K_{1}\right)} \cdots z_{\ell}^{N\left(K_{\ell}\right)}\right]=\exp \left\{\sum_{\emptyset \neq J \subseteq L} \mathbf{E} \Lambda\left(\bigcap_{j \in J}\left(K_{j} \oplus\left(-\Xi_{0}\right)\right)\right) \prod_{j \in J}\left(z_{j}-1\right)\right\}
$$

where the sum $\sum_{\emptyset \neq J \subseteq L}$ runs over all $2^{\ell}-1$ non-empty (unordered) subsets $J$ of $L$. In other words, $\left(N\left(K_{1}\right), \ldots, N\left(K_{\ell}\right)\right)$ possesses an $\ell$-variate Poisson distribution with parameters

$$
\mu_{J}=\mu_{\Lambda, Q}\left(K_{J}\right):=\mathbf{E} \Lambda\left(\bigcap_{j \in J}\left(K_{j} \oplus\left(-\Xi_{0}\right)\right)\right) \quad \text { for } \quad \emptyset \neq J \subseteq L
$$

and the consistency relations (1.6) are fulfilled with $\gamma_{J}=\gamma_{\Lambda, Q}\left(K_{J}\right)$ defined by

$$
\gamma_{\Lambda, Q}\left(K_{J}\right):=\mathbf{E} \Lambda\left(\bigcap_{j \in J}\left(K_{j} \oplus\left(-\Xi_{0}\right)\right)\right)-\mathbf{E} \Lambda\left(\bigcap_{j \in J}\left(K_{j} \oplus\left(-\Xi_{0}\right)\right) \cap \bigcup_{j \in L \backslash J}\left(K_{j} \oplus\left(-\Xi_{0}\right)\right)\right)
$$


for $\emptyset \subseteq J \subseteq L$

Proof. For each copy $\Xi_{i}$ of the typical grain $\Xi_{0}$ and any compact set $K \neq \emptyset$, it is evident that

$$
K \cap\left(\Xi_{i}+X_{i}\right) \neq \emptyset \quad \text { iff } \quad X_{i} \in K \oplus\left(-\Xi_{i}\right)
$$

Therefore, using the indicator function $\mathbf{1}_{B}(x)$ ( $=1$ for $x \in B$ and $=0$ otherwise) we may write $N(K)$ as sum

$$
N(K)=\#\left\{i \geq 1: K \cap\left(\Xi_{i}+X_{i}\right) \neq \emptyset\right\}=\sum_{i \geq 1} \mathbf{1}_{K \oplus\left(-\Xi_{i}\right)}\left(X_{i}\right)
$$

Hence, for $z_{1}, \ldots, z_{\ell} \in \mathbb{C}^{1}$ we may express the PGF of $\left(N\left(K_{1}\right), \ldots, N\left(K_{\ell}\right)\right)$ as follows:

$$
\mathbf{E}\left[z_{1}^{N\left(K_{1}\right)} \cdots z_{\ell}^{N\left(K_{\ell}\right)}\right]=\mathbf{E}\left[\prod_{i \geq 1}\left(z_{1}^{\mathbf{1}_{K_{1} \oplus\left(-\Xi_{i}\right)}\left(X_{i}\right)} \cdots z_{\ell}^{\mathbf{1}_{K_{\ell} \oplus\left(-\Xi_{i}\right)}\left(X_{i}\right)}\right)\right] .
$$

Next, we make use of the probability generating functional $G_{\Lambda, Q}[\mathrm{v}]=\mathbf{E}\left[\prod_{i \geq 1} \mathrm{v}\left(X_{i}, \Xi_{i}\right)\right]$ of an independently marked Poisson process $\Pi_{\Lambda, Q}=\sum_{i \geq 1} \delta_{\left[X_{i}, \Xi_{i}\right]}$ on $\mathbb{R}^{d} \times \mathcal{K}^{d}$ with intensity measure $\Lambda$ and mark distribution $Q . G_{\Lambda, Q}[\mathrm{v}]$ possesses a comparatively simple shape, see e.g. [16] (p. $65)$ or [2], namely,

$$
G_{\Lambda, Q}[\mathrm{v}]=\exp \left\{\int_{\mathbb{R}^{d}} \int_{\mathcal{K}^{d}}(\mathrm{v}(x, K)-1) Q(\mathrm{~d} K) \Lambda(\mathrm{d} x)\right\}
$$

for any Borel-measurable function $\mathrm{v} \mid \mathbb{R}^{d} \times \mathcal{K}^{d} \longrightarrow \mathbb{C}^{1}$ satisfying $\int_{\mathbb{R}^{d}} \int_{\mathcal{K}^{d}}|\mathrm{v}(x, K)-1| Q(\mathrm{~d} K) \Lambda(\mathrm{d} x)<\infty$.

In the particular case $\mathrm{v}(x, K)=\prod_{j=1}^{\ell} z_{j}^{\mathbf{1}_{K_{j} \oplus(-K)}(x)}$ the latter condition is an immediate consequence of (1.2). By applying the obvious identities $z^{\mathbf{1}_{B}(x)}=1+\mathbf{1}_{B}(x)(z-1)$ and

$$
\prod_{j \in L}\left(1+a_{j}\right)=1+\sum_{\emptyset \neq J \subseteq L} \prod_{j \in J} a_{i}
$$

for any $a_{1}, \ldots, a_{\ell} \in \mathbb{C}$, where the sum $\sum_{\emptyset \neq I \subseteq L}$ runs over all non-empty index sets $I \subseteq$ $\{1, \ldots, \ell\}$, we can express $\mathrm{v}(x, K)-1$ as follows:

$$
\mathrm{v}(x, K)-1=\prod_{j \in L}\left(1+\mathbf{1}_{K_{j} \oplus(-K)}(x)\left(z_{j}-1\right)\right)-1=\sum_{\emptyset \neq I \subseteq L} \prod_{i \in I}\left(\left(z_{i}-1\right) \mathbf{1}_{K_{i} \oplus(-K)}(x)\right) .
$$

By using the relation $\mathbf{1}_{A}(x) \mathbf{1}_{B}(x)=\mathbf{1}_{A \cap B}(x)$ and combined with a multiple application of 
Fubini's theorem we arrive at

$$
\begin{aligned}
\mathbf{E}\left[z_{1}^{N\left(K_{1}\right)} \cdots z_{\ell}^{N\left(K_{\ell}\right)}\right] & =\exp \left\{\sum_{\emptyset \neq J \subseteq L} \prod_{j \in J}\left(z_{j}-1\right) \int_{\mathbb{R}^{d}} \int_{\mathcal{K}_{d}} \prod_{j \in J} \mathbf{1}_{K_{i} \oplus(-K)}(x) Q(\mathrm{~d} K) \Lambda(\mathrm{d} x)\right\} \\
& =\exp \left\{\sum_{\emptyset \neq J \subseteq L} \prod_{j \in J}\left(z_{j}-1\right) \int_{\mathbb{R}^{d}} \int_{\mathcal{K}_{d}} \mathbf{1}_{j \in J} \bigcap_{j \in J}\left(K_{j} \oplus(-K)\right)(x) Q(\mathrm{~d} K) \Lambda(\mathrm{d} x)\right\} \\
& =\exp \left\{\sum_{\emptyset \neq J \subseteq L} \prod_{j \in J}\left(z_{j}-1\right) \mathbf{E} \Lambda\left(\bigcap_{j \in J}\left(K_{j} \oplus\left(-\Xi_{0}\right)\right)\right)\right\}
\end{aligned}
$$

The last line provides the asserted shape of the PGF (2.7).

To accomplish the proof of Theorem 2.1 we check the conditions (1.6) for $\mu_{J}$ given in (2.8). For notational ease we put $K_{i}^{0}:=K_{i} \oplus\left(-\Xi_{0}\right)$ for $i=1, \ldots, \ell$ and $\nu(\cdot)=\mathbf{E} \Lambda(\cdot)$. Applying the inclusion-exclusion formula

$$
\nu\left(A \cap \cup_{j \in L \backslash I} A_{j}\right)=\sum_{\emptyset \neq J \subseteq L \backslash I}(-1)^{\# J-1} \nu\left(A \cap \cap_{j \in J} A_{j}\right)
$$

(being valid for any additive set-function $\nu$ ) yields

$$
\begin{aligned}
\gamma_{\Lambda, Q}\left(K_{I}\right) & =\sum_{I \subseteq J \subseteq L}(-1)^{\#(J \backslash I)} \nu\left(\bigcap_{j \in J} K_{j}^{0}\right) \\
& =\nu\left(\bigcap_{i \in I} K_{i}^{0}\right)+\sum_{\emptyset \neq J \subseteq L \backslash I}(-1)^{\# J} \nu\left(\bigcap_{i \in I} K_{i}^{0} \cap \bigcap_{j \in J} K_{j}^{0}\right) \\
& =\nu\left(\bigcap_{i \in I} K_{i}^{0}\right)-\nu\left(\bigcap_{i \in I} K_{i}^{0} \cap \bigcup_{j \in L \backslash I} K_{j}^{0}\right) \geq 0 \quad \text { for } \quad \emptyset \neq I \subseteq L
\end{aligned}
$$

and, since $\mathbf{E} \Lambda\left(K_{i} \oplus\left(-\Xi_{0}\right)\right)>0$ for $i=1, \ldots, \ell$,

$$
\gamma_{\Lambda, Q}\left(K_{\emptyset}\right)=\sum_{\emptyset \neq J \subseteq L}(-1)^{\# J} \nu\left(\bigcap_{j \in J} K_{j}^{0}\right)=-\nu\left(\bigcup_{j \in L} K_{j}^{0}\right)<0 .
$$

This completes the proof of Theorem 2.1 .

In the particular case of a stationary BM $\Xi_{Q}^{\lambda}$ with known intensity $\lambda$ (see e.g. [13] for various methods to estimate the intensity $\lambda$ ) the parameters (2.8) contain quite a lot of information on the distribution $Q$ of the typical grain $\Xi_{0}$ depending on a clever choice of $K_{1}, \ldots, K_{\ell}$ (e.g. balls, line segments, single points etc.). For example, if $K_{i}=\left\{x_{i}\right\}$ for $i=1, \ldots, \ell$, the $2^{\ell}-1$ parameters $\mu_{Q}^{\lambda}\left(x_{J}\right):=\lambda \mathbf{E}\left|\bigcap_{j \in J}\left(\Xi_{0}-x_{j}\right)\right|_{d}$ for non-empty subsets $J$ of $L$ determine the joint distribution of the random vector $\left(N\left(\left\{x_{1}\right\}\right), \ldots, N\left(\left\{x_{\ell}\right\}\right)\right)$. The shift-invariance of the Lebesgue measure $|\cdot|_{d}$ causes that $\mu\left(x_{J}\right)$ remains unchanged when the points $\left(x_{j}\right)_{j \in J}$ is replaced by the shifted points $\left(x_{j}+x\right)_{j \in J}$ for any $x \in \mathbb{R}^{d}$. In particular, we have $\mu_{Q}^{\lambda}\left(x_{i}\right):=$ 
$\mathbf{E} N\left(\left\{x_{i}\right\}\right)=\lambda \mathbf{E}\left|\Xi_{0}\right|_{d}$ and

$$
\mu_{Q}^{\lambda}\left(x_{i j}\right):=\operatorname{Cov}\left(N\left(\left\{x_{i}\right\}\right), N\left(\left\{x_{j}\right\}\right)\right)=\lambda \mathbf{E}\left|\left(\Xi_{0}-x_{i}\right) \cap\left(\Xi_{0}-x_{j}\right)\right|_{d}=\lambda \mathbf{E} C_{\Xi_{0}}\left(x_{i}-x_{j}\right)
$$

for $i, j=1, \ldots, \ell$, where $C_{B}(x):=|B \cap(B+x)|_{d}$ denotes the set covariance function of the bounded Borel set $B \subset \mathbb{R}^{d}$, see e.g. [12] or [2] (p. 17) for properties and historical background.

Note that the $\ell$-point probabilities $\mathbf{P}\left(x_{1} \in \Xi_{Q}^{\lambda}, \ldots, x_{\ell} \in \Xi_{Q}^{\lambda}\right)$ can be completely expressed in terms of the parameters $\mu_{Q}^{\lambda}\left(x_{J}\right)$ for $\emptyset \neq J \subseteq L$.

Indeed, by a twofold application of the inclusion-exclusion formula and

$$
\mathbf{P}\left(\bigcap_{j \in J}\left\{x_{j} \notin \Xi\right\}\right)=\mathbf{P}\left(\bigcap_{j \in J}\left\{N\left(\left\{x_{j}\right\}\right)=0\right\}\right)=\exp \left\{-\lambda \mathbf{E}\left|\bigcup_{j \in J}\left(\Xi_{0}-x_{j}\right)\right|_{d}\right\}
$$

we find that

$$
\begin{aligned}
\mathbf{P}\left(x_{1} \in \Xi_{Q}^{\lambda}, \ldots, x_{\ell} \in \Xi_{Q}^{\lambda}\right) & =\mathbf{P}\left(\bigcap_{i \in L}\left\{N\left(\left\{x_{i}\right\}\right) \geq 1\right\}\right)=1-\mathbf{P}\left(\bigcup_{i \in L}\left\{N\left(\left\{x_{i}\right\}\right)=0\right\}\right) \\
& =1+\sum_{\emptyset \neq J \subseteq L}(-1)^{\# J} \exp \left\{-\lambda \mathbf{E}\left|\bigcup_{j \in J}\left(\Xi_{0}-x_{j}\right)\right|_{d}\right\}
\end{aligned}
$$

with $\lambda \mathbf{E}\left|\bigcup_{j \in J}\left(\Xi_{0}-x_{j}\right)\right|_{d}=\sum_{\emptyset \neq I \subseteq J}(-1)^{\# I-1} \mu_{Q}^{\lambda}\left(x_{I}\right)$.

Especially, the covariance of a stationary BM $\Xi_{Q}^{\lambda}$ takes the form

$$
\mathbf{P}\left(\mathbf{o} \in \Xi_{Q}^{\lambda}, x \in \Xi_{Q}^{\lambda}\right)=1-2 \exp \left\{-\lambda \mathbf{E}\left|\Xi_{0}\right|_{d}\right\}+\exp \left\{-2 \lambda \mathbf{E}\left|\Xi_{0}\right|_{d}+\lambda \mathbf{E} C_{\Xi_{0}}(x)\right\} .
$$

\section{Parameter estimation for stationary Boolean models}

The estimation of the $2^{\ell}-1$ parameters associated with the $\ell$-variate Poisson distribution of $\left(N\left(K_{1}\right), \ldots, N\left(K_{\ell}\right)\right)$ is possible in case of a stationary BM $\Xi_{Q}^{\lambda}$ when an observation in some sufficiently large (expanding) sampling window $W_{n}$ is available. For $\Lambda(\cdot)=\lambda|\cdot|_{d}$ the parameters (2.8) take the form

$$
\mu_{Q}^{\lambda}\left(K_{J}\right)=\lambda \mathbf{E}\left|\bigcap_{j \in J}\left(\Xi_{0} \oplus\left(-K_{j}\right)\right)\right|_{d} \quad \text { for } \quad \emptyset \neq J \subseteq L .
$$

An estimator for $\mu_{Q}^{\lambda}\left(K_{J}\right)$ is constructed from the empirical volume fractions

$$
\left(\widehat{p\left(K_{I}\right)}\right)_{n}:=\frac{\left|\left(\Xi_{Q}^{\lambda} \oplus \bigcup_{i \in I}\left(-K_{i}\right)\right) \cap W_{n}\right|_{d}}{\left|W_{n}\right|_{d}} \text { for } \quad \emptyset \neq I \subseteq J .
$$


Note that the numerator in (3.13) can be calculated only if $\Xi_{Q}^{\lambda}$ is known in the larger window $W_{n} \oplus \bigcup_{i \in I} K_{i}$. Otherwise, the mere observation of $\Xi_{Q}^{\lambda} \cap W_{n}$ means that in (3.13) $W_{n}$ must be replaced by the smaller minus-window $W_{n} \ominus \bigcup_{i \in I} K_{i}$, where $A \ominus B:=\{x: x+A \subseteq B\}$, see [13] (p. 58). In this case ${\widehat{p\left(K_{I}\right)}}_{n}$ is still unbiased and strongly consistent.

Theorem 3.1. Let $\Xi_{Q}^{\lambda}$ be a stationary $B M$ in $\mathbb{R}^{d}$ with intensity $\lambda>0$ and typical grain $\Xi_{0}$ having distribution $Q$ such that $\mathbf{E}\left|\Xi_{0} \oplus B_{r}^{d}\right|_{d}<\infty$ for $r>0$. Further, let $\left(W_{n}\right)_{n \geq 1}$ be an isotone sequence of convex and compact sets in $\mathbb{R}^{d}$ such that $\varrho\left(W_{n}\right):=\sup \left\{r>0: B_{r}^{d}+x \subseteq W_{n}\right.$ for some $\left.x \in W_{n}\right\} \underset{n \rightarrow \infty}{\longrightarrow} \infty$. Then, for any compact sets $K_{1}, \ldots, K_{\ell}$ as in Theorem 2.1 and any non-empty index set $J \subseteq L$, the sequence of estimators

$$
\left.\left(\mu_{Q}^{\lambda\left(K_{J}\right.}\right)\right)_{n}:=\sum_{\emptyset \neq I \subseteq J}(-1)^{\# I} \log \left(1-\widehat{p\left(K_{I}\right)_{n}}\right)
$$

is strongly consistent for $\mu_{Q}^{\lambda}\left(K_{J}\right)$, i.e. $\left.\left(\widehat{\mu_{Q}^{\lambda}\left(K_{J}\right.}\right)\right)_{n} \underset{n \rightarrow \infty}{\stackrel{\mathbf{P}-\text { a.s. }}{\longrightarrow}} \mu_{Q}^{\lambda}\left(K_{J}\right)$ for $\emptyset \neq J \subseteq L$.

Proof. Obviously, the Minkowski sum of the BM (1.1) with a fixed set $K \in \mathcal{K}^{d}$, expressed by

$$
\Xi_{\Lambda, Q} \oplus(-K)=\bigcup_{x \in K}\left(\Xi_{\Lambda, Q}-x\right)=\bigcup_{i \geq 1}\left(\Xi_{i} \oplus(-K)+X_{i}\right)
$$

yields again a BM defined by the same Poisson process $\Pi_{\Lambda}$ and the new typical grain $\Xi_{0} \oplus(-K)$. The stationarity of the BM $\Xi_{Q}^{\lambda}\left(=\Xi_{\Lambda, Q}\right.$ with $\left.\Lambda(\cdot)=\lambda|\cdot|_{d}\right)$ implies that its volume fraction equals

$$
\mathbf{E}\left|\left(\Xi_{Q}^{\lambda} \oplus(-K)\right) \cap[0,1]^{d}\right|_{d}=\mathbf{P}\left(\mathbf{o} \in \Xi_{Q}^{\lambda} \oplus(-K)\right)=1-\exp \left\{-\lambda \mathbf{E}\left|\Xi_{0} \oplus(-K)\right|_{d}\right\},
$$

see [12], [4], [13] or [2].

The spatial ergodic theorem of Nguyen \& Zessin, see [14] ( and e.g. [13], [7] for its application to BMs ), provides the a.s. limit

$$
\begin{aligned}
\frac{\left|\left(\Xi_{Q}^{\lambda} \oplus(-K)\right) \cap W_{n}\right|_{d}}{\left|W_{n}\right|_{d}} \frac{\mathbf{P}-\text { a.s. }}{n \rightarrow \infty} & \mathbf{E}\left|\left(\Xi_{Q}^{\lambda} \oplus(-K)\right) \cap[0,1]^{d}\right|_{d} \\
& =1-\exp \left\{-\lambda \mathbf{E}\left|\Xi_{0} \oplus(-K)\right|_{d}\right\} .
\end{aligned}
$$

Note that $W_{n}$ can be replaced by $W_{n} \ominus K=\left\{x: x+K \subseteq W_{n}\right\}$ without changing the limit. Applying the latter relation to $K=\bigcup_{i \in I} K_{i}$ for any index set $I \subseteq J(\subseteq L)$ gives

$$
1-\left(\widehat{p\left(K_{I}\right)}\right)_{n} \underset{n \rightarrow \infty}{\stackrel{\mathbf{P}-\text { a.s. }}{\longrightarrow}} 1-p\left(K_{I}\right):=\exp \left\{-\lambda \mathbf{E}\left|\bigcup_{i \in I}\left(\Xi_{0} \oplus\left(-K_{i}\right)\right)\right|_{d}\right\}
$$


which together with the inclusion-exclusion formula

$$
\sum_{\emptyset \neq I \subseteq J}(-1)^{\# I-1} \mathbf{E}\left|\bigcup_{i \in I}\left(\Xi_{0} \oplus\left(-K_{i}\right)\right)\right|_{d}=\mathbf{E}\left|\bigcap_{j \in J}\left(\Xi_{0} \oplus\left(-K_{j}\right)\right)\right|_{d}
$$

completes the proof of Theorem 3.1.

Note that the estimators (3.14) are neither asymptotically unbiased nor mean-square consistent. This is due to the fact that the $\mathrm{BM} \Xi_{Q}^{\lambda} \oplus(-K)$ may completely cover the window $W_{n}$ with positive probability $p_{n}(\underset{n \rightarrow \infty}{\longrightarrow} 0)$ if $\mathbf{E}\left|\Xi_{0} \oplus K\right|_{d}>0$. On the other hand, the ( $\left.2^{\ell}-1\right)$-dimensional vector $\left(\widehat{\mu_{Q}^{\lambda}\left(K_{J}\right)}\right)_{n}$ indexed by non-empty $J \subseteq L$ is asymptotically normally distributed (as $n \rightarrow \infty)$. More precisely, we are able to prove the multivariate central limit theorem

$$
\left.\sqrt{\left|W_{n}\right|_{d}}\left(\left(\mu_{Q}^{\lambda\left(K_{J}\right.}\right)\right)_{n}-\mu_{Q}^{\lambda}\left(K_{J}\right)\right)_{J \subseteq L} \underset{n \rightarrow \infty}{\stackrel{\mathcal{D}}{\longrightarrow}} \mathcal{N}_{2^{\ell}-1}\left(\mathbf{o}, \Sigma_{Q}^{\lambda}\left(K_{L}\right)\right),
$$

that is, the left-hand side converges in distribution to a mean zero Gaussian vector in $\mathbb{R}^{2^{\ell}-1}$ with covariance matrix $\Sigma_{Q}^{\lambda}\left(K_{L}\right)$ provided the condition $\mathbf{E}\left|\Xi_{0} \oplus B_{r}^{d}\right|_{d}^{2}<\infty$ for $r>0$ (implying $(1.2))$ is satisfied. We omit the proof (3.16) and the calculation of the rather complicated form of $\Sigma_{Q}^{\lambda}\left(K_{L}\right)$. We only mention that the proof of (3.16) relies on the fact (by applying the mean value theorem and Slutsky's lemma) that the distributional limits of $\sqrt{\left|W_{n}\right|_{d}}(\log (1-$ $\left.\left.\left(\widehat{p\left(K_{I}\right)}\right)_{n}\right)-\log \left(1-p\left(K_{I}\right)\right)\right)$ for $\emptyset \neq I \subseteq L$ coincides with the Gaussian limit

$$
\left.\sqrt{\left|W_{n}\right|_{d}}\left(\widehat{\left(\overline{p(K I}_{I}\right)}\right)_{n}-p\left(K_{I}\right)\right) /\left(p\left(K_{I}\right)-1\right) \underset{n \rightarrow \infty}{\stackrel{\mathcal{D}}{\longrightarrow}} \mathcal{N}\left(0, \sigma_{Q}^{\lambda}\left(K_{I}\right)\right)
$$

where the variance $\left.\sigma_{Q}^{\lambda}\left(K_{I}\right)=\lim _{n \rightarrow \infty}\left|W_{n}\right|_{d} \operatorname{Var}\left(\widehat{\left(p\left(K_{I}\right)\right.}\right)_{n}\right) /\left(1-p\left(K_{I}\right)\right)^{2}$ is equal to

$$
\int_{\mathbb{R}^{d}}\left(\exp \left\{\lambda \mathbf{E}\left|\left(\Xi_{0} \oplus\left(-\bigcup_{i \in I} K_{i}\right)\right) \cap\left(\Xi_{0} \oplus\left(-\bigcup_{i \in I} K_{i}\right)-x\right)\right|_{d}\right\}-1\right) \mathrm{d} x
$$

see [6] and [1], [15] for similar computations in connection with the proof of asymptotic normality of empirical Boolean model characteristics. Finally, to prove (3.16) we employ the Cramér-Wold technique which means to determine the distributional limits of linear combinations of the left-hand side of (3.17).

\section{Extensions to Other Poisson-Driven Random Sets}

To generalize Theorem 2.1 we make again use of the probability generating functional

$$
G_{\Lambda_{s}, Q}[\mathrm{w}]:=\mathbf{E}\left[\prod_{i \geq 1} \mathrm{w}\left(Y_{i}, M_{i}\right)\right]=\exp \left\{\int_{\mathbb{R}^{s}} \int_{\mathbb{M}}(\mathrm{w}(x, m)-1) Q(\mathrm{~d} m) \Lambda_{s}(\mathrm{~d} x)\right\}
$$


of the independently marked Poisson process $\Pi_{\Lambda_{s}, Q}=\sum_{i \geq 1} \delta_{\left[Y_{i}, M_{i}\right]}$ on $\mathbb{R}^{s} \times \mathbb{M}$ for some $s \geq 1$ with intensity measure $\Lambda_{s}$ on $\left[\mathbb{R}^{s}, \mathcal{B}\left(\mathbb{R}^{s}\right)\right]$ and mark distribution $Q$ on the Borel sets $\mathcal{B}(\mathrm{M})$ of a Polish mark space $\mathbb{M}$. Further, let $F \mid \mathbb{R}^{s} \times \mathbb{M} \rightarrow \mathcal{F}^{d}$ be a $\left(\mathcal{B}\left(\mathbb{R}^{s}\right) \otimes \mathcal{B}(\mathbb{M}), \sigma_{f}\right)$-measurable mapping, where $\mathcal{F}^{d}$ denotes the family of closed subsets in $\mathbb{R}^{d}$ equipped with Matheron's $\sigma$-algebra, $\sigma_{f}$ being a Borel- $\sigma$-algebra with respect to the hit-and-miss (or Fell) topology, see [12], [16]. Under the assumption that the number $N_{F}(K):=\#\left\{i \geq 1: K \cap F\left(Y_{i}, M_{i}\right) \neq\right.$ $\emptyset\}$ of members of the countable family $\left\{F\left(Y_{i}, M_{i}\right)\right\}_{i \geq 1}$ (forming a particle process) hitting $K$ has finite expectation for any $K \in \mathcal{K}^{d}$ (which implies the closedness of the union set $\left.\Xi_{\Lambda_{s}, Q}(F):=F\left(Y_{1}, M_{1}\right) \cup F\left(Y_{2}, M_{2}\right) \cup \cdots\right)$, we can show that the random $\mathbb{Z}_{+}^{\ell}$-valued vector $\left(N_{F}\left(K_{1}\right), \ldots, N_{F}\left(K_{\ell}\right)\right)$ possesses an $\ell$-variate Poisson distribution. To express the corresponding $2^{\ell}-1$ parameters we need the Borel sets $A(K, m):=\left\{y \in \mathbb{R}^{s}: K \cap F(y, m) \neq \emptyset\right\}$ for $K \in \mathcal{K}^{d}$ and $m \in \mathbb{M}$.

Theorem 4.1. Let the family $\left\{F\left(Y_{i}, M_{i}\right)\right\}_{i \geq 1}$ of random closed sets in $\mathbb{R}^{d}$ and the numbers $N_{F}(K)$ for $K \in \mathcal{K}^{d}$ defined as before. Then the $P G F$ of the random vector $\left(N_{F}\left(K_{1}\right), \ldots, N_{F}\left(K_{\ell}\right)\right)$ with fixed $K_{1}, \ldots, K_{\ell} \in \mathcal{K}^{d}$ such that $0<\mathbf{E} \Lambda_{s}\left(A\left(K_{j}, M_{0}\right)\right)<\infty$ for $i=1, \ldots, \ell$ takes the form (1.5) with parameters

$$
\mu_{J}=\mathbf{E} \Lambda_{s}\left(\bigcap_{j \in J} A\left(K_{j}, M_{0}\right)\right) \quad \text { for } \quad \emptyset \neq J \subseteq L
$$

The proof of Theorem 4.1 is omitted since it consists in repeating the arguments used to prove (2.7) up to some obvious changes. On the other hand, Theorem 2.1 is a special case of Theorem 4.1 with $\Lambda_{d}=\Lambda$ and $F(x, m)=m+x$ for $x \in \mathbb{R}^{d}, m \in \mathcal{K}^{d}$ so that $A(K, m)=K \oplus(-m)$. By choosing the mapping $F(x, m)$ and the mark space $\mathbb{M}$ appropriately we can count, for example, the number of strips of $2 D$-Poisson strip process, see Fig. 2, hitting a fixed planar compact set $K$. We close this section by describing this procedure for general (stationary) Poisson $k$-cylinder (and $k$-flat) processes in $\mathbb{R}^{d}, 1 \leq k \leq d-1$.

For doing this, some further notation is needed. In stochastic geometry, a $k$-cylinder in $\mathbb{R}^{d}$ is defined as Minkowski sum $\mathbb{L} \oplus B$ of a direction space $\mathbb{L} \in \mathcal{G}(d, k)$ (= the Grassmannian of $k$-dimensional subspaces of $\mathbb{R}^{d}$ ) and a compact base $B$ in the orthogonal complement $\mathbb{L}^{\perp}$, see e.g. [16] or [17]. In the following we go along the line suggested in [8] (which slightly differs from that in [12] and [17]) and identify $\mathbb{L}$ with a unique element $O_{\mathbb{L}}$ of the equivalence class $\mathbf{O}_{\mathbb{L}}$ of special orthogonal matrices $O \in \mathbb{S O}_{d}$ (i.e. $O \in \mathbb{R}^{d \times d}, O^{T}=O^{-1}$ and $\operatorname{det}(O)=1$ ) satisfying $O \mathbb{E}_{k}=\mathbb{L}$ (and $O \mathbb{E}_{k}^{\perp}=\mathbb{L}^{\perp}$ ), where $\mathbb{E}_{k}=\operatorname{span}\left\{e_{d-k+1}, \ldots, e_{d}\right\}, \mathbb{E}_{k}^{\perp}=\operatorname{span}\left\{e_{1}, \ldots, e_{d-k}\right\}$ for $k=1, \ldots, d-1$ with the usual orthonormal basis $\left\{e_{1}, \ldots, e_{d}\right\}$ of $\mathbb{R}^{d}$. In other words, two matrices $O_{1}, O_{2} \in \mathbb{S O}_{d}$ belong to the compact set $\mathbf{O}_{\mathbb{L}} \subset \mathbb{S O}_{d}$ iff $O_{1}^{T} O_{2}$ belongs to the set of 
orthogonal block matrices $\mathbb{S}\left(\mathbb{O}_{d-k} \times \mathbb{O}_{k}\right)$ defined by

$$
\left\{\left(\begin{array}{cc}
A & 0 \\
0 & B
\end{array}\right): A \in \mathbb{R}^{(d-k) \times(d-k)}, B \in \mathbb{R}^{k \times k}, A^{T}=A^{-1}, B^{T}=B^{-1}, \operatorname{det}(A)=\operatorname{det}(B)\right\} .
$$

The element $O_{\mathbb{L}}$ can be chosen in a canonical way, e.g. as lexicographically smallest element of the set of matrices $\mathbf{O}_{\mathbb{L}}$. In this way we get a one-to-one correspondence between $\mathbb{S O}_{k}^{d}=\left\{O_{\mathbb{L}}:=\right.$ lexmin $\left.\mathbf{O}_{\mathbb{L}}: \mathbb{L} \in \mathcal{G}(d, k)\right\}$ and $\mathcal{G}(d, k)$ up to orientation of the subspaces. Note that for $k=1$ (and analogously for $k=d-1$ ) the orthogonal matrix $O_{\mathbb{L}}$ is chosen such that $\operatorname{det}\left(O_{\mathbb{L}}\right)=1$ and $O_{\mathbb{L}} e_{d}=u$, where $u \in \partial B_{1}^{d}$ can be expressed in terms of spherical coordinates and $u$ and $-u$ are identified. Thus, for $d=2, k=1$ we represent $\mathbb{L}=\left\{\varrho(\cos \vartheta, \sin \vartheta)^{T}: \varrho \in \mathbb{R}^{1}\right\}$ by the matrix $O_{\mathbb{L}}=\left(\begin{array}{cc}\sin \vartheta & \cos \vartheta \\ -\cos \vartheta & \sin \vartheta\end{array}\right)$ for $0 \leq \vartheta<\pi$.

In this way, to each random subspace $\mathbb{L} \in \mathcal{G}(d, k)$ corresponds a (unique) random matrix $\Theta(\mathbb{L}) \in \mathbb{S O}_{k}^{d}$ and vice versa. Now, we are ready to define a Poisson $k$-cylinder process in $\mathbb{R}^{d}$ over some probability space $[\Omega, \mathcal{F}, \mathbf{P}]$ as countable family of random $k$-cylinders

$$
\left\{\Theta_{i}\left(\left(\left\{\left(x+Y_{i}, \mathbf{o}_{k}\right)^{T}: x \in \Xi_{i}\right\}\right) \oplus \mathbb{E}_{k}\right), i \geq 1\right\}=\left\{\Theta_{i}\left(\left(\Xi_{i}+Y_{i}\right) \times \mathbb{R}^{k}\right), i \geq 1\right\}
$$

driven by an independently marked Poisson process $\Pi_{\Lambda_{d-k}, Q_{d, k}}=\sum_{i \geq 1} \delta_{\left[Y_{i},\left(\Theta_{i}, \Xi_{i}\right)\right]}$ on the product space of $\mathbb{R}^{d-k}$ and mark space $\mathbb{S O}_{k}^{d} \times \mathcal{K}^{d-k}$ with intensity measure $\Lambda_{d-k}$ and typical mark $\left(\Theta_{0}, \Xi_{0}\right)$ (specifying direction and base of the typical $k$-cylinder $\Theta_{0}\left(\Xi_{0} \times \mathbb{R}^{k}\right)$ ) with distribution $Q_{d, k}$. Note that, in analogy to (1.2), the condition $\mathbf{E} \Lambda_{d-k}\left(B_{r}^{d-k} \oplus\left(-\Xi_{0}\right)\right)<\infty$ for any $r>0$ implies the closedness of the Poisson $k$-cylinder model (= union set of the $k$-cylinders (4.20), see also [8]. Furthermore, a Poisson $k$-cylinder process resp. model is stationary iff $\Lambda_{d-k}(\cdot)=\lambda|\cdot|_{d-k}$.

It is easy to see that Poisson $k$-cylinder processes fit within the framework of Theorem 4.1. Namely, for $s=d-k, Q=Q_{d, k}$ and $F(y,(\theta, \xi)):=\theta\left((\xi+y) \times \mathbb{R}^{k}\right)$ for $y \in \mathbb{R}^{d-k}$ and $(\theta, \xi) \in \mathbb{M}:=\mathbb{S O}_{k}^{d} \times \mathcal{K}^{d-k}$, we find that

$$
\begin{aligned}
A(K,(\theta, \xi)) & =\left\{y \in \mathbb{R}^{d-k}: K \cap F(y,(\theta, \xi)) \neq \emptyset\right\} \\
& =\pi_{d-k}\left(\theta^{T} K\right) \oplus(-\xi) \text { for } \quad K \in \mathcal{K}^{d},
\end{aligned}
$$

where $\pi_{d-k}(x)$ denotes the projection of the vector $x \in \mathbb{R}^{d}$ on its first $d-k$ components.

Hence, the corresponding parameters (4.19) for Poisson $k$-cylinder processes are as follows:

$$
\mu_{J}=\mathbf{E} \Lambda_{d-k}\left(\bigcap_{j \in J}\left(\pi_{d-k}\left(\Theta_{0}^{T} K_{j}\right) \oplus\left(-\Xi_{0}\right)\right)\right) \quad \text { for } \quad \emptyset \neq J \subseteq L
$$


In the particular case of stationary Poisson $k$-flat processes in $\mathbb{R}^{d}$, where $\Xi_{0}=\left\{\mathbf{o}_{d-k}\right\}$ and $\Lambda_{d-k}(\cdot)=\lambda|\cdot|_{d-k}$, the parameters (4.21) take the form

$$
\mu_{J}=\lambda \mathbf{E}\left|\bigcap_{j \in J} \pi_{d-k}\left(\Theta_{0}^{T} K_{j}\right)\right|_{d-k} \quad \text { for } \quad \emptyset \neq J \subseteq L
$$

\section{References}

[1] Böhm, S., Heinrich, L. And Schmidt, V. (2004). Asymptotic properties of estimators for the volume fractions of jointly stationary random sets. Statistica Neerlandica 58, 388406.

[2] Chiu, S. N., Stoyan, D., Kendall, W.S. and Mecke, J. (2013). Stochastic Geometry and Its Applications. 3rd ed., Wiley, Chichester.

[3] Dwass, M., Teicher, H. (1957) On infinitely divisible random vectors. Ann. Math. Stat. 28, No. 2, 461 - 470 .

[4] Hall, P. (1988). Introduction to the Theory of Coverage Processes. Wiley, New York.

[5] HeinRich, L. (1992). On existence and mixing properties of germ-grain models. Statistics 23, 271-286.

[6] Heinrich, L., Molchanov, I. (1999) Central limit theorem for a class of random measures associated with germ-grain models. Adv. Appl. Prob. 31, No. 2, 283 - 314.

[7] Heinrich, L. (2005). Large deviations of the empirical volume fraction for stationary Poisson grain models. Ann. Appl. Prob. 15, No. 1A, 392-420.

[8] Heinrich, L., Spiess, M. (2013) Central limit theorems for volume and surface content of stationary Poisson cylinder processes in expanding domains. Adv. Appl. Prob. 45, No. $2,312-331$.

[9] Johnson, N. L., Kotz, S. and Balakrishnan, N. (1997). Discrete Multivariate Distributions. Wiley, Chichester.

[10] Kawamura, K. (1979). The structure of the multivariate Poisson distribution. Kodai Mathematical Journal 2, 337-345.

[11] Kawamura, K. (1987). Calculation of the density for the multivariate Poisson distribution. Kodai Mathematical Journal 10, 231-241.

[12] Matheron, G. (1975). Random Sets and Integral Geometry. Wiley, New York. 
[13] Molchanov, I. (1997). Statistics of the Boolean Model for Practitioners and Mathematicians. Wiley, Chichester.

[14] Nguyen, X.X. And Zessin, H. (1979). Ergodic theorems for spatial processes. Z. Wahrsch. verw. Gebiete 48, 133-158.

[15] Schmidt, V., Spodarev, E. (2005) Joint estimators for the specific intrinsic volumes of stationary random sets. Stoch. Proc. Appl. 115, 959 - 981.

[16] Schneider, R. And WeIL, W. (2008). Stochastic and Integral Geometry. Springer, Berlin.

[17] Spiess, M. and Spodarev, E. (2011). Anisotropic Poisson processes of cylinders. Methodol. Comput. Appl. Probab. 13, 801-819. 